\title{
Identification of three extra-chromosomal replicons in Leptospira pathogenic strain and development of new shuttle vectors
}

\author{
Weinan Zhu' ${ }^{1}$ Jin Wang ${ }^{2}$, Yongzhang Zhu', Biao Tang ${ }^{3}$, Yunyi Zhang ${ }^{2}$, Ping He${ }^{1}$, Yan Zhang ${ }^{1}$, Boyu Liu', \\ Xiaokui Guo ${ }^{1 *}$, Guoping Zhao ${ }^{2,3^{*}}$ and Jinhong Qin ${ }^{1 *}$
}

\begin{abstract}
Background: The genome of pathogenic Leptospira interrogans contains two chromosomes. Plasmids and prophages are known to play specific roles in gene transfer in bacteria and can potentially serve as efficient genetic tools in these organisms. Although plasmids and prophage remnants have recently been reported in Leptospira species, their characteristics and potential applications in leptospiral genetic transformation systems have not been fully evaluated.

Results: Three extrachromosomal replicons designated Icp1 (65,732 bp), Icp2 (56,757 bp), and Icp3 (54,986 bp) in the L. interrogans serovar Linhai strain 56609 were identified through whole genome sequencing. All three replicons were stable outside of the bacterial chromosomes. Phage particles were observed in the culture supernatant of 56609 after mitomycin C induction, and Icp3, which contained phage-related genes, was considered to be an inducible prophage. L. interrogans-Escherichia coli shuttle vectors, constructed with the predicted replication elements of single rep or rep combined with parAB loci from the three plasmids were shown to successfully transform into both saprophytic and pathogenic Leptospira species, suggesting an essential function for rep genes in supporting auto-replication of the plasmids. Additionally, a wide distribution of homologs of the three rep genes was identified in L. interrogans isolates, and correlation tests showed that the transformability of the shuttle vectors in L. interrogans isolates depended, to certain extent, on genetic compatibility between the rep sequences of both plasmid and host.

Conclusions: Three extrachromosomal replicons co-exist in L. interrogans, one of which we consider to be an inducible prophage. The vectors constructed with the rep genes of the three replicons successfully transformed into saprophytic and pathogenic Leptospira species alike, but this was partly dependent on genetic compatibility between the rep sequences of both plasmid and host.
\end{abstract}

Keywords: Leptospira interrogans, Plasmid, Prophage, Genetic transformation systems

\section{Background}

Leptospirosis is one of the most widespread zoonoses caused by infection with a group of pathogenic spirochetes, primarily various strains of the species Leptospira interrogans [1,2]. Since the first complete genome of the bacterium was published a decade ago [3], remarkable

\footnotetext{
* Correspondence: xkguo@shsmu.edu.cn; gpzhao@sibs.ac.cn; jinhongqin@sjtu.edu.cn

'Department of Microbiology and Immunology, Institutes of Medical Science, Shanghai Jiao Tong University School of Medicine, 280 South Chongqing Road, Shanghai 200025, China

${ }^{2}$ CAS Key Laboratory of Synthetic Biology, Institute of Plant Physiology and Ecology, Shanghai Institute for Biological Sciences, Chinese Academy of Sciences, Shanghai, China

Full list of author information is available at the end of the article
}

progress has been made in understanding its genetic blueprint as well as the functions of a variety of its genes. However, major obstacles in the genetic analysis of $L$. interrogans remain; these are partly related to the slow growth rate of the bacterium and the lack of efficient genetic manipulation tools [4-6].

Plasmids and prophages are known to contribute to horizontal gene transfer, and their remnants are commonly found in bacterial genomes [7-11]. Because these elements can carry diverse genetic information allowing them to play specific physiological roles in the host bacterium, they potentially serve as efficient genetic tools [12-16]. Numerous linear and circular plasmids 
have been found in another pathogenic spirochete, Borrelia burgdorferi, and nine of them were identified as being prophage derived [17-21]. Among them, pGK12 was developed as an expression vector for B. burgdorferi, and this plasmid has significantly assisted investigation of the in vivo functions of particular genes [22-24].

In the genus Leptospira, saprophytic Leptospira biflexa serovar Patoc strain Patoc I was shown to harbor a plasmid (P74) and atemperate phage, LE1 [25-28]. The latter, which was developed as a L. biflexa-Escherichia coli shuttle vector, contains the LE1 replication region and an antibiotic resistance marker, and has been shown to replicate in saprophytic $L$. biflexa, but not in pathogenic L. interrogans [27]. Although plasmids and prophage remnants have recently been reported in other Leptospira species [29], a leptospiral genetic transform system has not yet been well established. Recently, random transposon mutagenesis and targeted mutagenesis in pathogenic leptospires have been achieved, and these have undoubtedly assisted genetic characterization of potential virulence factors in pathogenic $L$. interrogans [5,6,30-32]. A method for conjugative transfer between E. coli and Leptospira spp. has also been developed using RP4 derivative conjugative plasmids to deliver the transposon, Himar1 [4]. However, genetic complementation of knockout mutants remains a challenge and still has to be conducted using transposon-mediated insertion or homologous recombination. Because the transposon integration site is uncertain, substantial time and energy are required to verify successful complementation. Therefore, development of an efficient genetic manipulation system in pathogenic Leptospira remains a highly warranted research goal.

In this study, we sequenced the complete genome of the highly virulent $L$. interrogans serogroup Grippotyphosa serovar Linhai strain 56609, followed by an intensive and detailed study of the three extrachromosomal circular replicons that were identified, with the goal of obtaining better understanding of this bacterium.

\section{Results}

Complete sequencing and de novo assembly of the $L$. interrogans serovar Linhai strain 56609 genome revealed three extrachromosomal circular DNA replicons

Three circular extrachromosomal replicons, designated lcp1, lcp2, and lcp3 were found in L. interrogans serovar Lai strain 56609 through whole genome sequencing and de novo assembly (Figure 1). According to the level of sequence-read coverage in each assembly, it was estimated that the three replicons shared equal copy numbers with the chromosomes. The overall $\mathrm{G}+\mathrm{C}$ contents of lcp1 and lcp2 were, at about 35\%, similar to that of the chromosomes, whereas lcp3 had a relatively higher $\mathrm{G}+\mathrm{C}$ content of $39 \%$. Basic Local Alignment Search
Tool (BLAST) analysis suggested that the vast majority of the genes encoded by the three replicons were hypothetical proteins, while a small portion encoded phage-related proteins (Additional file 1: Table S1). It is noteworthy that lcp3 encoded more phage-related proteins $(22,73.3 \%$ of the total 30 coding sequences with assigned functions) than the other two extrachromosomal replicons, lcp1 $(9,29.0 \%$ of the total 31 coding sequences with assigned functions) and lcp2 (6, 20\% of the total 30 coding sequences with assigned functions). Furthermore, the strand-bias distribution of the lcp3 genes, with 71 out of 77 genes clustered on the same strand was also significantly different from that of the other two replicons.

\section{Plasmid DNA extraction followed by physical mapping confirmed the auto-replication traits of Icp1, Icp2, and Icp3}

To confirm that the three putative low-copy-number circular plasmid replicons (lcp1, lcp2 and lcp3) were definitely extrachromosomal replicons, plasmid DNA from $L$. interrogans serovar Linhai strain 56609 was extracted using the classical alkaline lysis method and was then used for Southern blot analysis employing both plasmidderived and chromosomal DNA-specific probes. As indicated in Additional file 2, in addition to the untreated DNA extracts, a specific restriction endonuclease, which has a unique restriction site in each of the candidate plasmids, was used to digest the DNA extracts separately in order to observe their linear configurations. DNA bands corresponding to different configurations of the plasmids were apparent after hybridization with plasmidspecific probes but absent using the chromosomal flaB specific probe. This result suggested that the three replicons were all present in the bacteria as circular extrachromosomal plasmids.

To determine whether the three plasmids were integrate into chromosomes, genomic DNA was digested with selected rare-cutting restriction enzymes followed by in situ pulsed-field gel electrophoresis (PFGE)-based Southern blot analysis. For lcp3, cutting with the restriction enzymes NotI and $A s c \mathrm{I}$ (each has a single restriction site in lcp3), was confirmed by Southern blotting using an lcp3-specific probe. The result showed that DNA bands of about $50 \mathrm{~kb}$ were obtained, a result consistent with the in silico predicted size of $\mathrm{lcp} 3$ (Figure 2A). Similarly, after digestion with a single restriction enzyme and Southern blot hybridization with lcp1- and lcp2specific probes, the same restriction patterns were observed for lcp1 and lcp2; these were identical to the linearized forms of these plasmids (Figure $2 \mathrm{~B}$ and $\mathrm{C}$ ). When the extracted genomes containing the replicons were analyzed by PFGE without digestion, no linear forms of the replicons were detected by Southern blots, 


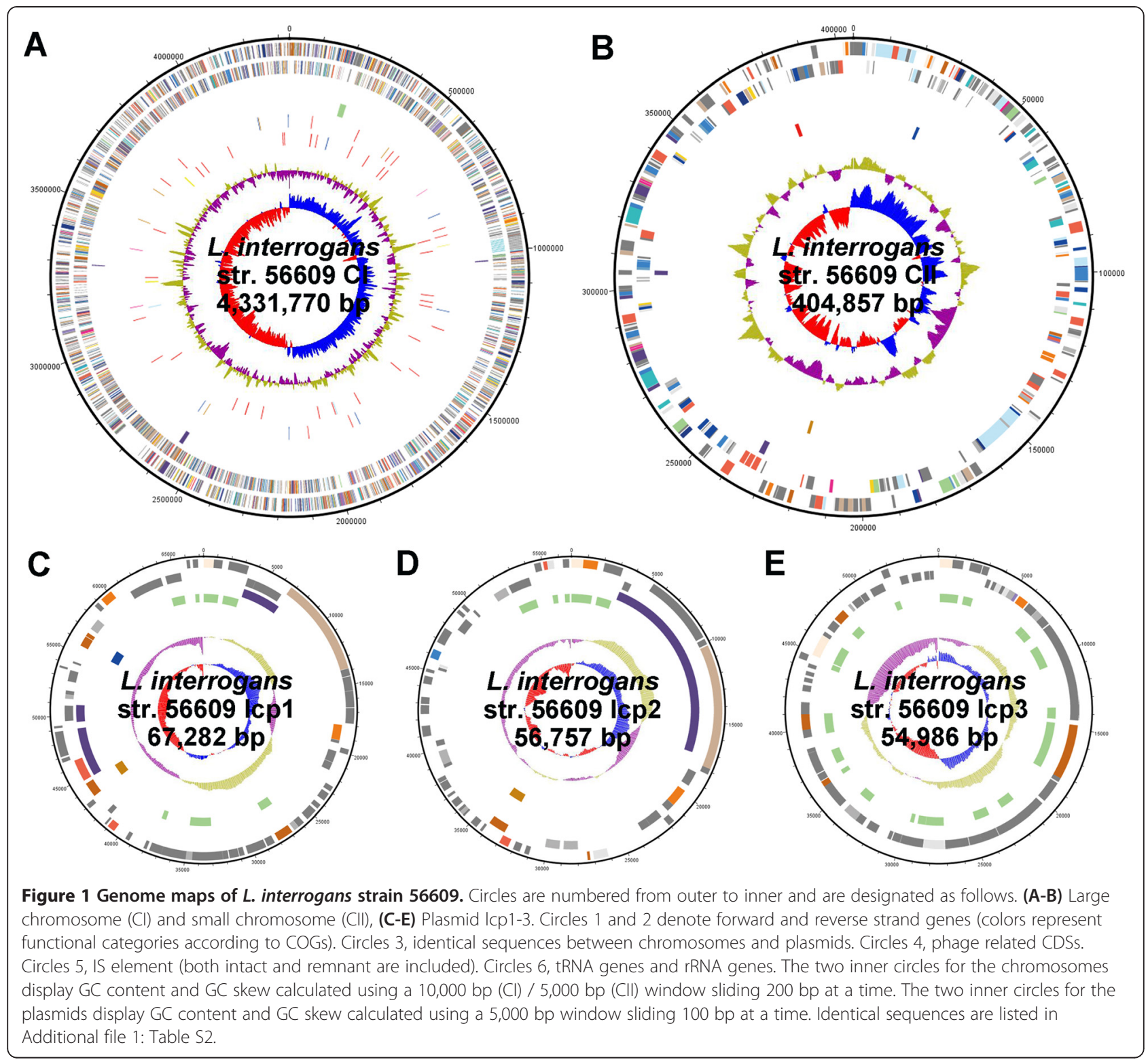

and the visible bands after ethidium bromide staining possibly represented remnants of the degraded genomes.

During sequence assembly, we found five sequences that were identical among the lcp1 and lcp2 plasmids and the chromosomes listed in Additional file 1: Table S2. To confirm the circular nature of lcp1 and lcp2, and to also exclude the possibility of plasmid integration, polymerase chain reaction (PCR) strategies employing primer pairs encompassing identical sequences between the plasmids and chromosomes were amplified, followed by DNA sequencing of the PCR products. Figure 3 shows the PCR results encompassing the large identical sequence repeats 5 (rp5) (13.7 kb) between lcp2 and chromosome CI as well as the identical repeat sequence rp2 $(2.3 \mathrm{~kb})$ between lcp1 and chromosome CI. PCR products were successfully obtained with the plasmid-located primer pairs (i.e. P2.1 and P2.2, P1.1 and P1.2), as were products from chromosomally located primer pairs (i.e. C1 and C2, C3 and C4), thereby confirming that lcp1 and lcp2 are extrachromosomal. The assumption that the two plasmids might be capable of chromosomal integration was rejected when no PCR products from plasmid-located primer and chromosomally located primer pairs were detected (e.g. P1.1 and C4, P1.2 and C3, P2.1 and C2, P2.2 and C1), thus excluding the possibility of lcp1 and lcp2 integrating into the chromosomes (Figure 3).

Taken together, these results show that the three plasmids are extrachromosomal, and also exclude the possibility that they might be able to integrate into chromosomes. In addition, PFGE analysis of strain 

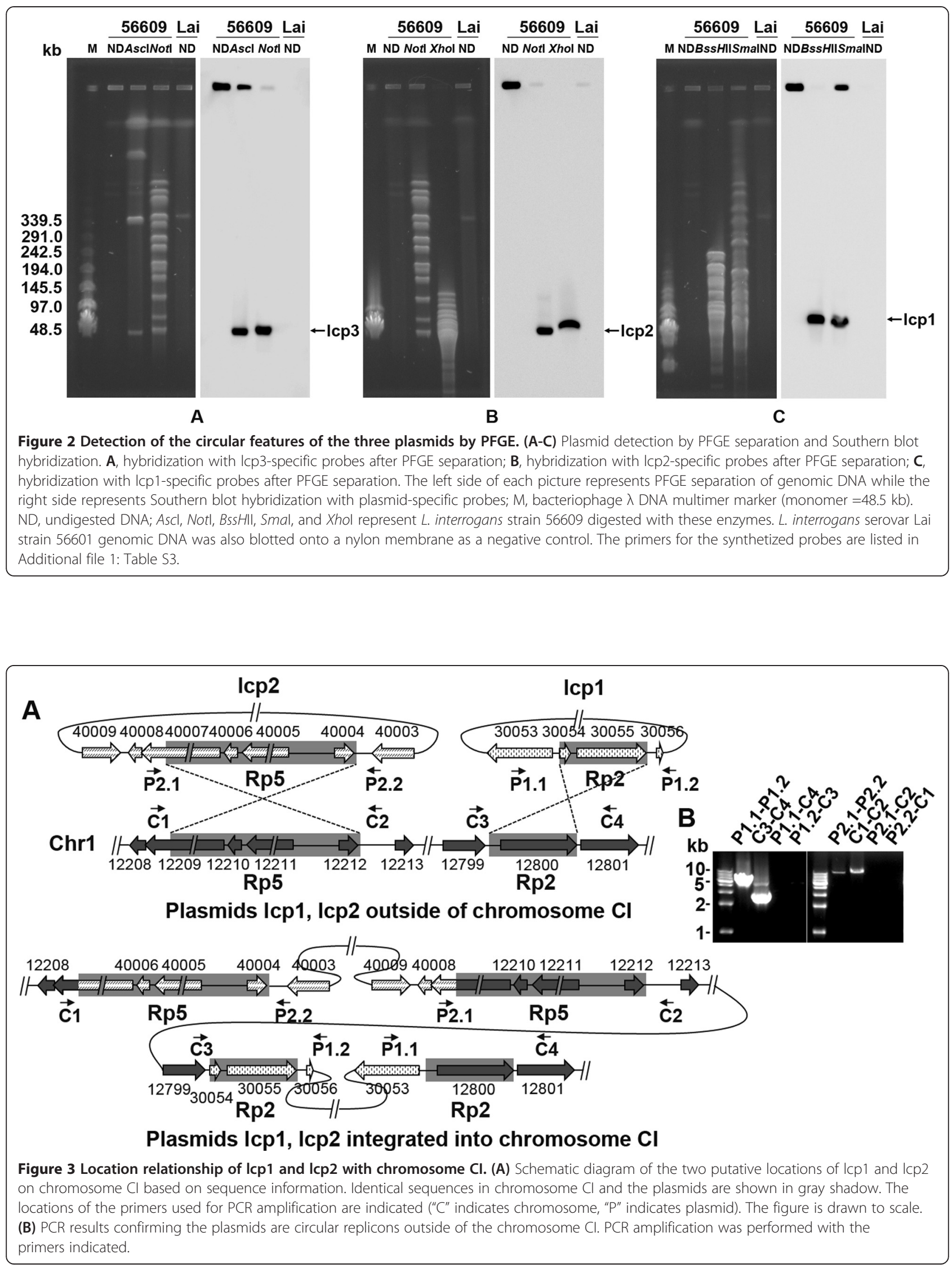
56609', which originated from strain 56609 after prolonged in vitro laboratory passage, digested with same single restriction enzymes, showed identical bands as those from strain 56609, which further confirms the high stability of these circular plasmids (Additional file 3).

\section{Plasmid Icp3 is likely to be an inducible prophage in $L$.} interrogans strain 56609

According to the annotation results, plasmid lcp3 encodes 30 phage-related proteins, including those for bacteriophage replication, regulation, packaging, head and tail morphogenesis, and lytic cycle-related proteins; however, it lacks integrase and is, therefore, thought to be a prophage. Mitomycin $C$ was used to induce prophage production. Two days after addition of mitomycin C to L. interrogans strain 56609 cultures, cell lysis was apparent by a decrease in the optical density of the culture (Figure 4A); lysis was nearly complete in 8 days, and was confirmed by dark-field microscopy. Transmission electron microscopy examination revealed bacteriophage-like particles (Figure 4B), each with a 45-50 $\mathrm{nm}$ diameter isometric head and an approximately 90-nm-long noncontractile tail, both of which are morphologically similar to those of the Siphoviridae family. Additionally, phage particles of the same size were observed inside L. interrogans cells $12 \mathrm{~h}$ after addition of mitomycin C (Figure 4C).

Expression of all 60 phage-related genes in lcp1, lcp2, and lcp3 was examined by reverse-transcription (RT)PCR after mitomycin $\mathrm{C}$ induction. With the exception of the open reading frames (ORFs) LIL50001 to LIL50003, which are involved in the partition and replication of plasmid lcp3 and are expressed at all stages, all of the other phage-related genes in lcp3 were specifically activated at different times by mitomycin $\mathrm{C}$ induction. Meanwhile, most ORFs in lcp1 and lcp2 showed no obvious changes in expression after mitomycin $C$ induction (Additional file 4A). Seven representative genes in lcp3 were chosen for further examination by quantitative (q)PCR, and similar results were obtained (Additional file $4 \mathrm{~B})$. These results, to some extent, suggest that plasmid lcp3 is likely to be an inducible prophage in $L$. interrogans strain 56609.

\section{Characterization of replication origins in the three} plasmids and development of a transformation system for pathogenic Leptospira species

Plasmid partition systems usually consist of the two partition proteins called ParA and ParB; these constitute the partition complex and are encoded by two coupled
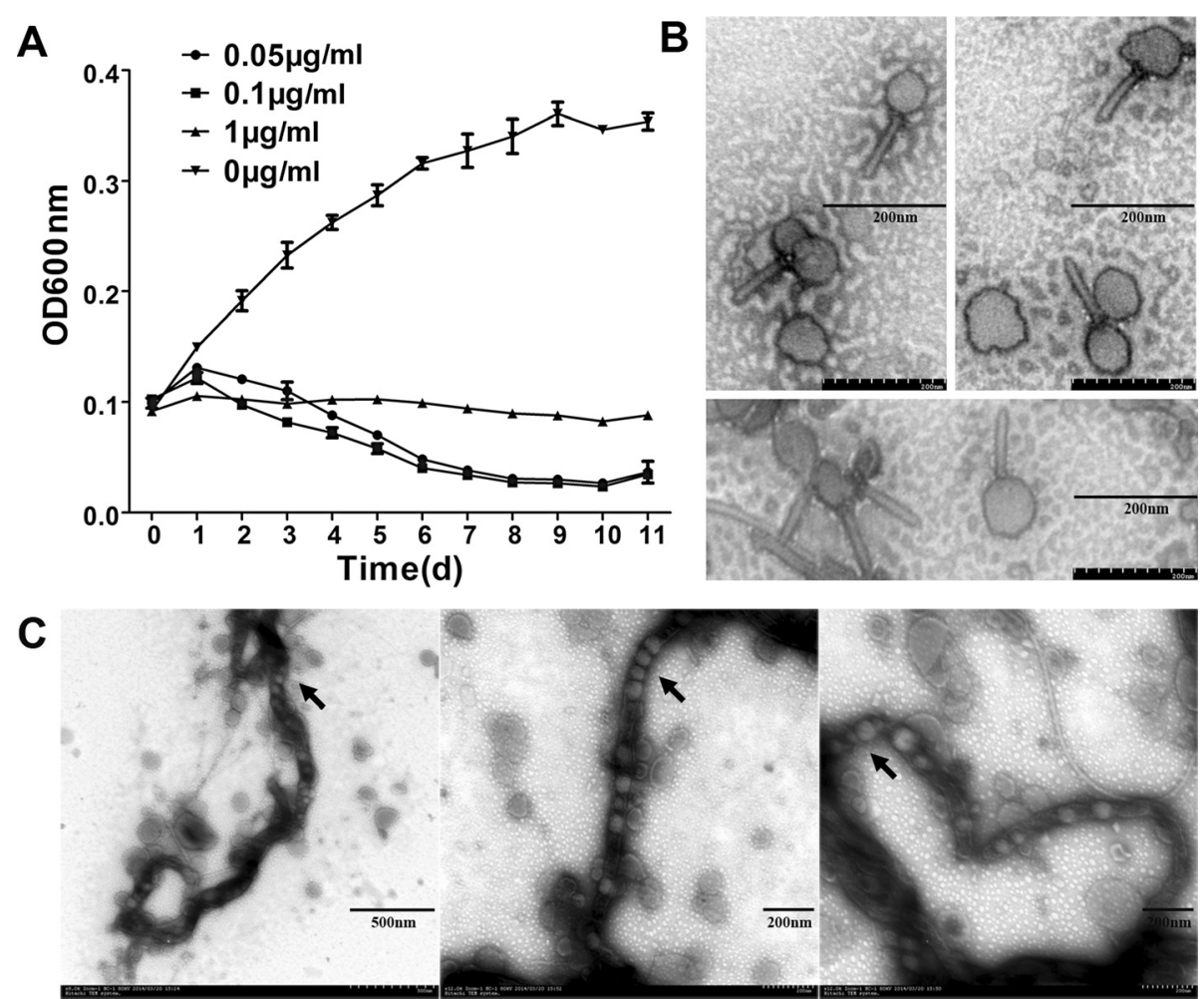

Figure 4 Phage particle induction from L. interrogans strain 56609. (A) Leptospira growth curve induced by different concentrations mitomycin C. Experiments were performed in triplicate. (B) Transmission electron microscopy photograph of phage particles. bar, $200 \mathrm{~nm}$. (C) Transmission electron microscopy photograph of phage particles inside a Leptospira cell. bar (left), $500 \mathrm{~nm}$, bar (middle and right), $200 \mathrm{~nm}$. 
genes known as $\operatorname{par} A B[33,34]$. Bioinformatic analysis revealed parAB genes in the three plasmids (i.e. LIL30001 and LIL30002 in lcp1, LIL40001 and LIL40002 in lcp2, and LIL50001 and LIL50002 in lcp3). Furthermore, rep genes encoding replication proteins in the three plasmids (i.e., LIL30003 in lcp1, LIL40003 in lcp2, and LIL50003 in lcp3) were found to be located immediately downstream of par $A B$ in their corresponding plasmids. All of these Rep proteins contain putative helix-turn-helix motifs, similar to Orf5 encoded by the LE1 phage genome in $L$. biflexa [27], and LA1839 encoded by the genomic island LaiGI in the L. interrogans serovar Lai genome [35]. LIL50003 in lcp3 exhibited low sequence similarity with Orf5 with only $27 \%$ identity and $73 \%$ coverage, whereas LIL40003 in lcp2 was highly similar to LA1839 with 96\% identity and $96 \%$ coverage.

To characterize the essential replication regions in the three plasmids, DNA fragments containing single rep or rep combined with parAB loci were PCR amplified and then cloned into the $L$. biflexa-E. coli shuttle vector (pGKBLe24) to replace the original LE1 rep gene. Six $L$. interrogans-E. coli shuttle vectors were thus generated and designated lcp1L and lcp1S, lcp2L and lcp2S, and lcp3L and lcp3S, containing either rep and parAB in "L" or rep alone in "S", respectively. The plasmids prepared from E. coli were transformed into different Leptospira strains by electroporation. Transformed strains included the pathogenic L. interrogans strains 56601, 56606 and 56610, and the saprophytic L. biflexa strain Patoc I. The LE1 rep-based shuttle vector pGKBLe24 was used as a positive control and its rep deleted pGKBLe24-rep derivative was used as a negative control (Figure 5A). As expected, the positive control (pGKBLe24) was able to transform into saprophytic L. biflexa but was unable to transform into pathogenic Leptospira species [27]. Shuttle vectors lcp3L/S from lcp3 were able to transform into all four of the Leptospira strains we tested. However, the shuttle vectors lcp1L/S from lcp1, could transform into the 56601 and 56610 strain, while the shuttle vectors lcp2L/S from lcp2 could only transform into the saprophytic L. biflexa Patoc I strain. All transformants were confirmed by PCR using primers specific for the shuttle vectors (Figure $5 \mathrm{~B}$ ). The plasmid transformation efficiencies for the saprophytic $L$. biflexa and pathogenic L. interrogans strains were similar, reaching $5 \times 10^{-6}$ electrotransformants per recipient cell. The stability of the shuttle vectors in $L$. interrogans cells was confirmed by a hamster infection model and PCR amplification of the shuttle vector genes in pathogenic $L$. interrogans recovered from the livers of the infected hamsters (Figure $5 \mathrm{C}$ and $\mathrm{D}$ ). Our data not only confirmed autonomous replication capability for all three plasmids, but also revealed the differential transformability of the shuttle vector constructs with different replication origins against various kinds of recipient hosts, as summarized in Table 1.

To detect if differences in host-specificity resulted from genetic incompatibility in the hosts, we tested 15 Leptospira strains endemic in China for the presence of rep genes from the three plasmids using Southern blot hybridization (Additional file 5). Comparisons of the transformability data against the rep sequence distribution for the three plasmids from strains 56601, 56606 and 56610 showed that the shuttle vectors only transformed hosts lacking the rep sequence of the same origin. Therefore, genetic incompatibility is likely to account for the differences observed in the shuttle vectors abilities to transform into different Leptospira species recipients.

Finally, Rep protein from the three plasmids studied herein appears to be widely distributed among Leptospira strains, as shown by NCBI nonredundant protein database searches on Leptospira species genome using BLASTp, where 49 hits for lcp1, 22 hits for lcp2, and seven hits for lcp3 (at least 75\% query coverage and 75\% identity) were obtained. This finding again suggests that complete sequencing and de novo assembly are critical for novel discoveries from genomic data mining.

\section{Discussion}

Extrachromosomal replicons were identified in pathogenic L. interrogans in this study. Because of the high proportion of homologous sequences among lcp1, lcp2 and chromosomes, plasmids tend to be easily assembled into chromosomes. Additionally, the low copy number of plasmids makes it hard to differentiate them from the large number of sequence reads generated from chromosomes. Furthermore, low sequence similarity among proteins encoded by extrachromosomal replicons also makes it difficult to identify plasmid-specific proteins based on sequence comparisons against other wellcharacterized plasmids. Also, the large sizes of plasmids also challenges methods designed to verify auto-replicons in bacteria. In this study, we confirmed the location of all three replicons in L. interrogans through the combined use of genomic sequence-directed PCR, plasmid-specific probe-based Southern hybridization and extrachromosomal replicon-oriented PFGE. All the results indicated the stable presence of the three plasmids as auto-replicons in the bacterium. Furthermore, we observed phage particles in L. interrogans strain 56609 cultures by induction with mitomycin $C$, a well-known inducer of phages. Genomic annotation identified parA, parB, and rep homologous sequences in lcp1, lcp2, and lcp3, all of which are notable for their indispensable roles in plasmid replication and stability $[33,34,36,37]$. The function of the three replicons was verified also by constructing functional shuttle vectors capable of autonomous replication in $L$. biflexa and/or $L$. interrogans. The results confirmed that these 


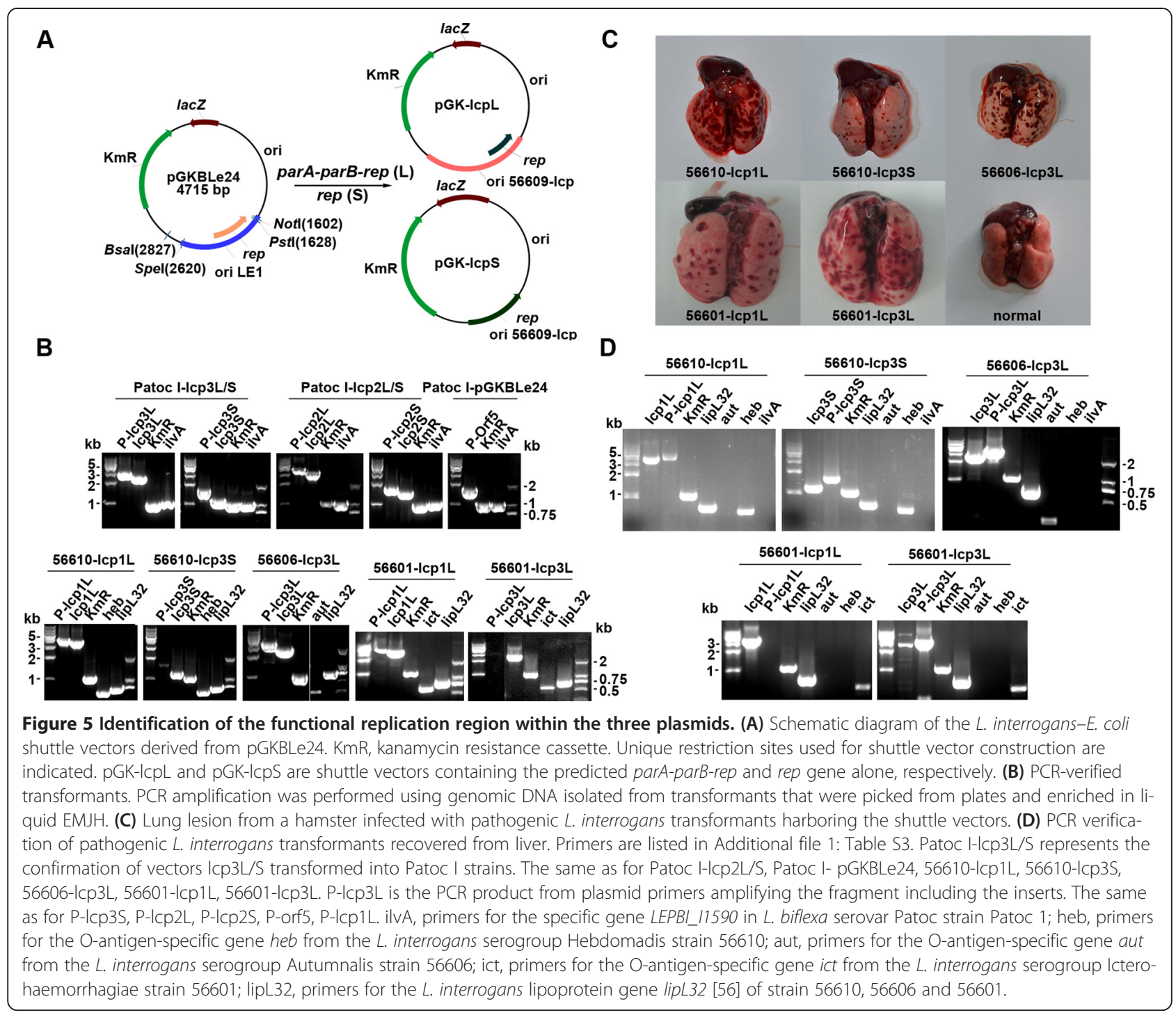

replicons are extra-chromosomally present and able to be auto-replicated.

For the purpose of genetic manipulation, electroporation employing suicide delivery vectors is the first choice for most researchers. When transposons are constructed in a suicide delivery vector, the transformation efficiency is about $5.1 \times 10^{-9}$ electrotransformants per recipient cell for $L$. interrogans and $2 \times 10^{-7}$ for $L$. biflexa
[4]. Conjugative plasmids delivering the Himar1 transposon between E. coli and Leptospira spp. reached efficiencies of $8.5 \times 10^{-8}$ transconjugants per recipient cell in L. interrogans and $1 \times 10^{-6}$ in L. biflexa, respectively, values around 10 times higher than that of transformation. Although these established genetics systems facilitate the manipulation of functional genes in Leptospira, particularly in saprophytic L. biflexa, genetic

Table 1 Transformation efficiency of shuttle vectors constructed in this study in four Leptospira strains

\begin{tabular}{|c|c|c|c|c|c|c|c|c|}
\hline \multirow[t]{2}{*}{ Leptospira strains } & \multirow[t]{2}{*}{ Icp1L/S } & \multirow[t]{2}{*}{ Icp2L/S } & \multirow[t]{2}{*}{ Icp3L/S } & \multicolumn{3}{|c|}{ pGKBLe24-rep } & \multirow[t]{2}{*}{$\mathrm{ddH}_{2} \mathrm{O}$} & \multirow[t]{2}{*}{ pGKBLe24 } \\
\hline & & & & Icp1 & Icp2 & Icp3 & & \\
\hline L. interrogans serovar Autumnalis str. 56606 & - & - & + & - & - & - & - & - \\
\hline L. interrogans serovar Hebdomadis str. 56610 & + & - & + & - & - & - & - & - \\
\hline L. interrogans serovar Lai str. 56601 & + & - & + & - & - & - & - & - \\
\hline L. biflexa serovar Patoc str. Patoc I & - & + & + & - & - & - & - & + \\
\hline L. interrogans serovar Linhai str. 56609 & - & - & - & - & - & - & - & - \\
\hline
\end{tabular}


manipulation in pathogenic L. interrogans remains especially difficult because of the low efficiencies of electroporation and conjugation combined with the slow growth rate of $L$. interrogans. In this study, the electroporation transformation efficiency of the replication plasmids reached $5 \times 10^{-6}$ electrotransformants per recipient cell in both $L$. biflexa and L. interrogans. This indicates that the transformation efficiency of these newly constructed replicative plasmids in L. interrogans is 100 to 1000 times higher than before.

Combining the transformability data from the shuttle vectors $(\mathrm{lcp} 1 \mathrm{~L} / \mathrm{S}, \mathrm{lcp} 2 \mathrm{~L} / \mathrm{S}$ and $1 \mathrm{cp} 3 \mathrm{~L} / \mathrm{S})$, with the wide distribution of the rep gene in different hosts, suggests that genetic incompatibility between plasmids and hosts should be considered in Leptospira strains because shuttle vectors apparently cannot transform into a host that harbors the same rep gene. However, a plasmid's inability to be transformed cannot be fully attributed to genetic incompatibility between itself and its host. Although Orf5 is absent in L. interrogans, it is reported that a shuttle vector containing Orf5 is unable to transform into pathogenic Leptospira strains [27,28,35]. We have also confirmed that it was unable to transform into $L$. interrogans strains 56601, 56606 and 56610. It has been reported that the shuttle vector derived from the Rep protein LA1839 in LaiGI can transform into L. interrogans Hond Utrecht IV and L. biflexa Patoc I strains but is unable to transform the L. interrogans Copenhageni strain [35]. Meanwhile, the rep gene $L A \_1839$ was found to be present in the L. interrogans Hond Utrecht IV strain but not in the L. interrogans Copenhageni strain. Hence, further systematic analyses are needed to elucidate the mechanism(s) underlying genetic transformability among Leptospira species, especially for pathogenic $L$. interrogans strains.

\section{Conclusions}

Research on pathogenic Leptospira has lagged far behind that of other pathogenic bacteria, primarily because of the lack of effective genetic manipulation tools suitable for L. interrogans. In fact, the first major breakthrough in understanding the genetics of $L$. interrogans came with the availability of its whole genome sequence $[3,38,39]$ and accumulation of abundant genomic sequencing data from it. With comprehensive information about the genetic blue print of $L$. interrogans coupled with epigenetic readouts from transcriptomics and proteomics in this species, in vitro biochemical and molecular biological analyses have already unveiled some of the molecular mechanisms underlying the physiology and pathology of this bacterium. However, without access to an efficient genetic manipulation system for in vivo analysis, it is impossible to obtain comprehensive knowledge about the biology of $L$. interrogans through in vitro biochemical analysis and sequence data based on in silico analysis alone. Therefore, the shuttle vectors developed and validated in this study represent a new milestone in research on pathogenic Leptospira. Moreover, these resources have strong potential to improve our understanding of the genomic composition of $L$. interrogans and genetic incompatibility in this bacterium.

\section{Methods}

\section{Bacteria}

L. interrogans serogroup Grippotyphosa serovar Linhai strain 56609 was initially isolated from a human patient with leptospirosis in Linhai, Zhejiang Province in 1954, and was maintained by the Chinese Center for Disease Control and Prevention (China CDC). Its virulence was preserved by serial passages in guinea pigs. The corresponding avirulent strain 56609' is derived from strain 56609 and has lost its virulence after more than 5 years of in vitro passages in our laboratory. Saprophytic $L$. biflexa serovar Patoc strain Patoc I, pathogenic Leptospira interrogans serovar Lai strain 56601, L. interrogans serovar Autumnalis strain 56606 and L. interrogans serovar Hebdomadis strain 56610 were also from China CDC. All the strains were grown in liquid EllinghausenMcCullough-Johnson-Harris (EMJH) medium under aerobic conditions at $28^{\circ} \mathrm{C}$, cultured to mid-exponential phase, and then collected for further analysis.

\section{Sequencing and assembly}

Five micrograms of 56609 genomic DNA was used for preparing sequencing libraries according to the manufacturer's protocol (454 Life Sciences, Roche). Sequencing was performed separately on two different 454 GS system platforms, one on the 454 GS 20 system and the other on the 454 GS FLX system [40-42]. In total, 554,443 reads were generated and 530,080 reads of high quality (96\%) were selected for genome assembly, providing 29.5 fold coverage. Phred, Phrap, and Consed programs were used for quality assessment and sequence assembly [43-45]. Altogether, we obtained 295 contigs (235 contigs $>500 \mathrm{bp}$ ), and the N50 size of the contigs was 20,058 bps. Contig order was determined by ContigScape plugin [46] and by referring to the reference genome sequence of the $L$. interrogans serovar Lai strain 56601 and the serovar Copenhageni strain L1-130. Physical gaps were closed by primer walking, PCR, and multiplex PCR methods. Finally, the error rate for strain 56609 was 0.4 errors per 10,000 bps. Genome validation was achieved by comparison of the in silico digestion results with in situ PFGE using rare-cutting restriction enzymes. The complete genomic sequences of $L$. interrogans strain 56609 have been deposited in GenBank under the following accession numbers: CP006723, CP006724, CP006725, CP006726, and CP006727. 


\section{Genome annotation}

ORFs were identified by Glimmer, GeneMark, and Z-curve programs with a further manual check applied [47-49]. Functional annotation was performed by comparison with L. interrogans serovar Lai and Copenhageni reference strains through the BLASTp program using the blast search criterion E-value of $10^{-3}$, whereby at least $75 \%$ of the smaller sequence was aligned. Clusters of orthologous groups (COG )functional classification for each gene was assigned using RPS-BLAST against the Conserved Domain Database (CDD), and domain analysis was performed by searching the Pfam database with an E-value of $10^{-3}$, which gave further verification and supplementation of the annotation [50,51]. If there were no BLAST hits, another BLASTp search was performed against the NCBI nonredundant protein database to determine the sequence similarity by removing aligned regions that were less than $75 \%$ of the smaller sequence. Transfer RNA genes were identified with tRNAscan-SE [52]. Insertion sequence (IS) elements were found by searching with the IS-finder online tool (https://www-is.biotoul.fr/) and by BLAST searches according to the reference sequences.

\section{DNA manipulation}

The classical alkaline lysis method was performed (with slight modification) to isolate large circular single-copy plasmids from $L$. interrogans strain 56609. Leptospira cells were resuspended in solution $1(50 \mathrm{mM}$ glucose, 10 mM EDTA pH 8.0, 25 mM Tris- $\mathrm{HCl}$ pH 8.0, $2.5 \mathrm{mg} /$ $\mathrm{ml}$ lysozyme) at $37^{\circ} \mathrm{C}$ for $1 \mathrm{~h}$. Solution $2(0.2 \mathrm{~N} \mathrm{NaOH}$, $1 \%$ SDS) was added and the solution incubated at $55^{\circ} \mathrm{C}$ for $30 \mathrm{~min}$. Solution 3 (5 M sodium acetate, $60 \mathrm{ml}$; acetic acid, $11.5 \mathrm{ml}$; sterilized water, $28.5 \mathrm{ml}$ per $100 \mathrm{ml}$ ) was added with thorough mixing, and then the solution was cooled on ice for $5 \mathrm{~min}$. After centrifugation, the supernatant was collected and extracted with acidic phenol-chloroform (NaAc-saturated phenol, $\mathrm{pH} 4.5$ : chloroform: isoamyl alcohol at 25: 24: 1) followed by $\mathrm{pH}$ neutral phenol-chloroform (Tris- $\mathrm{HCl}$ saturated phenol, pH 7.9: chloroform: isoamyl alcohol at 25: 24: 1). DNA was precipitated with isopropanol and washed twice with $70 \%$ ethanol. The purified DNA was dissolved in sterile deionized water and stored at $-20^{\circ} \mathrm{C}$.

For PFGE, the cells were embedded in agarose plugs and then lysed by protease treatment as previously described [53]. For digestion of DNA plugs, PFGE was performed in a contour-clamped homogeneous electric field DRII apparatus (Bio-Rad Laboratories). A program with ramping from 5 to $65 \mathrm{~s}$ for $20 \mathrm{~h}$ at $6 \mathrm{v} / \mathrm{cm}$ was employed to separate the DNA fragments. For Southern blot analysis, the digoxigenin-labeled probes were generated through PCR amplification using the oligonucleotide primers listed in Additional file 1: Table S3 (PCR DIG Probe Synthesis Kit, Roche). Hybridization and detection were performed with a DIG DNA detection system (Roche) according to the manufacturer's protocol.

\section{Template prophage induction and microscopy of phage particles}

A bacterial culture at early logarithmic growth phase was divided into four aliquots and a mitomycin $\mathrm{C}$ gradient at a final concentration of either $1 \mu \mathrm{g} / \mathrm{ml}, 100 \mathrm{ng} / \mathrm{ml}, 50 \mathrm{ng} / \mathrm{ml}$, or $0 \mathrm{ng} / \mathrm{ml}$, was added to each aliquot. Phage particles were collected after 4 days with $100 \mathrm{ng} / \mathrm{ml}$ mitomycin C added by centrifugation at $12,000 \times \mathrm{g}$ for $30 \mathrm{~min}$ twice to remove the residual bacteria followed by a final ultracentrifugation at $40,000 \times \mathrm{g}$ for $2 \mathrm{~h}$. The phage sample was stained with $2 \%$ phosphotungstic acid and examined on a Hitachi 700 transmission electron microscope.

\section{RNA isolation, reverse transcriptase polymerase chain reaction ( $R T-P C R$ ), and quantitative PCR (qPCR)}

Induced bacterial cells were harvested at various times after addition of $100 \mathrm{ng} / \mathrm{ml}$ of mitomycin C. RNA was extracted using TRIzol reagent (Invitrogen) according to the manufacturer's instructions and converted to cDNA using the Transcriptor First Strand cDNA Synthesis Kit (Roche). PCR primer pairs were designed for target ORFs in the three plasmids. Seven genes from lcp3 were selected for qPCR. For each amplification reaction, the $\mathrm{C}_{T}$ (threshold cycle) of the flaB gene from the same sample was used as an internal control to normalize the tested gene amplicon for $\mathrm{C}_{T}$ calculation, and the relative fold changes were calculated as described previously $[54,55]$.

\section{Plasmid construction and electroporation-mediated transformation of Leptospira species}

The nucleotide sequences of the three plasmid replication regions (single rep and rep combined with the $\operatorname{par} A B$ locus) were amplified with the primer pairs shown in Additional file 1: Table S3 and inserted into the L. biflexa-E. coli shuttle vector pGKBLe24 (a gift from Dr. Picardeau, Institute Pasteur, France) to replace the LE1 replication region and generate new plasmids, herein referred to as pGK-lcpL and pGK-lcpS. The pGKBLe24 $^{\text {-rep }}$ (LE1 replication region deletion with different restriction enzyme sites) of each three plasmids was self-ligated as a negative control. The new shuttle vectors and control plasmids were enriched from $E$. coli using a Plasmid Maxi Kit (QIAGEN). Leptospira cells were electrotransformed as described previously [27]. Kanamycin-resistant clones were picked from plates and transferred to $2 \mathrm{ml} \mathrm{EMJH}$ liquid media containing kanamycin and cultured until they had grown sufficiently. Further verification was achieved by genomic DNA isolation followed by PCR amplification with the primers listed in Additional file 1: Table S3 [56]. 


\section{Additional files}

Additional file 1: Table S1. General feature of L. interrogans serovar Linhai str. 56609 genome. Table S2. Identical sequences between plasmids Icp1 and Icp2 and chromosomes*. Table S3 Oligonucleotide primers used in this study. Table S4. Distribution of three plasmids in 15 Chinese epidemic Leptospira strains.

Additional file 2: Characterization of genome DNA from the three plasmids by agarose gel electrophoresis and Southern blotting.

Additional file 3: Stability of the three plasmids within the L. interrogans strain 56609.

Additional file 4: RT-PCR and qPCR to detect gene expression after induction.

Additional file 5: Identification of the three plasmids in 15 Chinese epidemic Leptospira strains and the saprophytic $L$. biflexa serovar Patoc strain Patoc I.

\section{Competing interests}

The authors declare that they have no competing interests.

\section{Authors' contributions}

$W Z, J Q, G Z$ and $X G$ designed the experiments; WZ, BT and $Y Z$ performed the experiments; JQ, GZ and XG designed the research; WZ, JW, JQ, GZ and XG wrote the manuscript. All authors discussed the results and commented on the manuscript. All authors read and approved the final manuscript.

\section{Acknowledgements}

This work was supported, in part, by the National Natural Science Foundation of China (grant numbers 81371771, 30970125, 81271793, $81201334,81261160321,81101264$ and 81171587), the Natural Science Foundation of Shanghai (grant number 132R1423000), the National High Technology Research and Development Program of China Program (grant 2011AA02A119). We thank HongliangYang for assistance with the preparation of the manuscript

\section{Author details}

${ }^{1}$ Department of Microbiology and Immunology, Institutes of Medical Science, Shanghai Jiao Tong University School of Medicine, 280 South Chongqing Road, Shanghai 200025, China. ${ }^{2}$ CAS Key Laboratory of Synthetic Biology, Institute of Plant Physiology and Ecology, Shanghai Institute for Biological Sciences, Chinese Academy of Sciences, Shanghai, China. ${ }^{3}$ State Key Laboratory of Genetic Engineering, Department of Microbiology, School of Life Sciences, Fudan University, 220 Handan Road, Shanghai 200433, China.

Received: 14 April 2014 Accepted: 4 February 2015

Published online: 15 February 2015

\section{References}

1. Evangelista KV, Coburn J. Leptospira as an emerging pathogen: a review of its biology, pathogenesis and host immune responses. Future Microbiol. 2010;5(9):1413-25.

2. Adler B, de la Pena Moctezuma A. Leptospira and leptospirosis. Vet Microbiol. 2010;140(3-4):287-96.

3. Ren SX, Fu G, Jiang XG, Zeng R, Miao YG, Xu H, et al. Unique physiological and pathogenic features of Leptospira interrogans revealed by wholegenome sequencing. Nature. 2003;422(6934):888-93.

4. Picardeau M. Conjugative transfer between Escherichia coli and Leptospira spp. as a new genetic tool. Appl Environ Microbiol. 2008;74(1):319-22.

5. Bourhy P, Louvel H, Saint Girons I, Picardeau M. Random insertional mutagenesis of Leptospira interrogans, the agent of leptospirosis, using a mariner transposon. J Bacteriol. 2005;187(9):3255-8

6. Ristow $P$, Bourhy $P$, da Cruz McBride FW, Figueira CP, Huerre $M$, Ave $P$, et al. The OmpA-like protein Loa22 is essential for leptospiral virulence. PLoS Pathog. 2007;3(7):e97.

7. Zhong L, Cheng Q, Tian X, Zhao L, Qin Z. Characterization of the replication, transfer, and plasmid/lytic phage cycle of the Streptomyces plasmid-phage pZL12. J Bacteriol. 2010;192(14):3747-54.
8. Hanna LF, Matthews TD, Dinsdale EA, Hasty D, Edwards RA. Characterization of the ELPhiS prophage from Salmonella enterica serovar Enteritidis strain LK5. Appl Environ Microbiol. 2012;78(6):1785-93.

9. Canchaya C, Desiere F, McShan WM, Ferretti JJ, Parkhill J, Brussow H. Genome analysis of an inducible prophage and prophage remnants integrated in the Streptococcus pyogenes strain SF370. Virology. 2002;302(2):245-58.

10. Skarin H, Hafstrom T, Westerberg J, Segerman B. Clostridium botulinum group III: a group with dual identity shaped by plasmids, phages and mobile elements. BMC Genomics. 2011;12:185.

11. Hargreaves KR, Colvin HV, Patel KV, Clokie JJ, Clokie MR. Genetically Diverse Clostridium difficile Strains Harboring Abundant Prophages in an Estuarine Environment. Appl Environ Microbiol. 2014;80(8):2644.

12. Zou QH, Li QH, Zhu HY, Feng Y, Li YG, Johnston RN, et al. SPC-P1: a pathogenicity-associated prophage of Salmonella paratyphi C. BMC Genomics. 2010;11:729.

13. Matos RC, Lapaque N, Rigottier-Gois L, Debarbieux L, Meylheuc T, Gonzalez-Zorn $B$, et al. Enterococcus faecalis prophage dynamics and contributions to pathogenic traits. PLOS Genet. 2013;9(6):e1003539

14. Tyler JS, Beeri K, Reynolds JL, Alteri CJ, Skinner KG, Friedman JH, et al. Prophage induction is enhanced and required for renal disease and lethality in an EHEC mouse model. PLoS Pathog. 2013;9(3):e1003236.

15. Eggers CH, Kimmel BJ, Bono JL, Elias AF, Rosa P, Samuels DS. Transduction by phiBB-1, a bacteriophage of Borrelia burgdorferi. J Bacteriol. 2001;183(16):4771-8.

16. Humphrey SB, Stanton TB, Jensen NS, Zuerner RL. Purification and characterization of $\mathrm{VSH}-1$, a generalized transducing bacteriophage of Serpulina hyodysenteriae. J Bacteriol. 1997;179(2):323-9.

17. Brisson D, Zhou W, Jutras BL, Casjens S, Stevenson B. Distribution of cp32 prophages among Lyme disease-causing spirochetes and natural diversity of their lipoprotein-encoding erp loci. Appl Environ Microbiol. 2013;79(13):4115-28

18. Eggers $\mathrm{CH}$, Samuels DS. Molecular evidence for a new bacteriophage of Borrelia burgdorferi. J Bacteriol. 1999;181(23):7308-13.

19. Zhang H, Marconi RT. Demonstration of cotranscription and 1-methyl-3nitroso-nitroguanidine induction of a 30-gene operon of Borrelia burgdorferi: evidence that the 32-kilobase circular plasmids are prophages. J Bacteriol. 2005;187(23):7985-95.

20. Casjens S, Palmer N, van Vugt R, Huang WM, Stevenson B, Rosa P, et al. A bacterial genome in flux: the twelve linear and nine circular extrachromosomal DNAs in an infectious isolate of the Lyme disease spirochete Borrelia burgdorferi. Mol Microbiol. 2000;35(3):490-516.

21. Eggers $\mathrm{CH}$, Casjens S, Hayes SF, Garon CF, Damman CJ, Oliver DB, et al Bacteriophages of spirochetes. J Mol Microbiol Biotechnol. 2000;2(4):365-73.

22. Sartakova M, Dobrikova E, Cabello FC. Development of an extrachromosomal cloning vector system for use in Borrelia burgdorferi. Proc Natl Acad Sci U S A. 2000;97(9):4850-5.

23. Sartakova ML, Dobrikova EY, Motaleb MA, Godfrey HP, Charon NW, Cabello FC. Complementation of a nonmotile flaB mutant of Borrelia burgdorferi by chromosomal integration of a plasmid containing a wild-type flaB allele. J Bacteriol. 2001;183(22):6558-64.

24. Sartakova ML, Dobrikova EY, Terekhova DA, Devis R, Bugrysheva JV, Morozova OV, et al. Novel antibiotic-resistance markers in pGK12-derived vectors for Borrelia burgdorferi. Gene. 2003;303:131-7.

25. Picardeau M, Bulach DM, Bouchier C, Zuerner RL, Zidane N, Wilson PJ, et al. Genome sequence of the saprophyte Leptospira biflexa provides insights into the evolution of Leptospira and the pathogenesis of leptospirosis. PLoS One. 2008;3(2):e1607

26. Saint Girons I, Margarita D, Amouriaux P, Baranton G. First isolation of bacteriophages for a spirochaete: potential genetic tools for Leptospira. Res Microbiol. 1990;141(9):1131-8.

27. Girons IS, Bourhy P, Ottone C, Picardeau M, Yelton D, Hendrix RW, et al. The LE1 bacteriophage replicates as a plasmid within Leptospira biflexa: construction of an L. biflexa-Escherichia coli shuttle vector. J Bacteriol. 2000;182(20):5700-5.

28. Bourhy P, Frangeul L, Couve E, Glaser P, Saint Girons I, Picardeau M. Complete nucleotide sequence of the LE1 prophage from the spirochete Leptospira biflexa and characterization of its replication and partition functions. J Bacteriol. 2005;187(12):3931-40.

29. Qin JH, Zhang Q, Zhang ZM, Zhong Y, Yang Y, Hu BY, et al. Identification of a novel prophage-like gene cluster actively expressed in both virulent and avirulent strains of Leptospira interrogans serovar Lai. Infect Immun. 2008;76(6):2411-9. 
30. Croda J, Figueira CP, Wunder Jr EA, Santos CS, Reis MG, Ko Al, et al. Targeted mutagenesis in pathogenic Leptospira species: disruption of the $\mathrm{LigB}$ gene does not affect virulence in animal models of leptospirosis. Infect Immun. 2008;76(12):5826-33.

31. Liao S, Sun A, Ojcius DM, Wu S, Zhao J, Yan J. Inactivation of the fliY gene encoding a flagellar motor switch protein attenuates mobility and virulence of Leptospira interrogans strain Lai. BMC Microbiol. 2009;9:253.

32. Murray GL, Morel V, Cerqueira GM, Croda J, Srikram A, Henry R, et al. Genome-wide transposon mutagenesis in pathogenic Leptospira species. Infect Immun. 2009;77(2):810-6.

33. Vecchiarelli AG, Han YW, Tan X, Mizuuchi M, Ghirlando R, Biertumpfel C, et al. ATP control of dynamic P1 ParA-DNA interactions: a key role for the nucleoid in plasmid partition. Mol Microbiol. 2010;78(1):78-91.

34. Schumacher MA, Funnell BE. Structures of ParB bound to DNA reveal mechanism of partition complex formation. Nature. 2005;438(7067):516-9.

35. Bourhy P, Salaun L, Lajus A, Medigue C, Boursaux-Eude C, Picardeau M. A genomic island of the pathogen Leptospira interrogans serovar Lai can excise from its chromosome. Infect Immun. 2007;75(2):677-83.

36. Sevastsyanovich YR, Titok MA, Krasowiak R, Bingle LE, Thomas CM. Ability of IncP-9 plasmid pM3 to replicate in Escherichia coli is dependent on both rep and par functions. Mol Microbiol. 2005;57(3):819-33.

37. Kwong SM, Yeo CC, Poh CL. Molecular analysis of the pRA2 partitioning region: ParB autoregulates parAB transcription and forms a nucleoprotein complex with the plasmid partition site, parS. Mol Microbiol. 2001:40(3):621-33.

38. Zhong Y, Chang X, Cao XJ, Zhang Y, Zheng H, Zhu Y, et al. Comparative proteogenomic analysis of the Leptospira interrogans virulence-attenuated strain IPAV against the pathogenic strain 56601. Cell Res. 2011:21(8):1210-29.

39. Nascimento AL, Verjovski-Almeida S, Van Sluys MA, Monteiro-Vitorello CB, Camargo LE, Digiampietri LA, et al. Genome features of Leptospira interrogans serovar Copenhageni. Braz J Med Biol Res. 2004;37(4):459-77.

40. Margulies M, Egholm M, Altman WE, Attiya S, Bader JS, Bemben LA, et al Genome sequencing in microfabricated high-density picolitre reactors. Nature. 2005;437(7057):376-80.

41. Droege M, Hill B. The Genome Sequencer FLX System-longer reads, more applications, straight forward bioinformatics and more complete data sets. J Biotechnol. 2008;136(1-2):3-10.

42. Gilles A, Meglecz E, Pech N, Ferreira S, Malausa T, Martin JF. Accuracy and quality assessment of 454 GS-FLX Titanium pyrosequencing. BMC Genomics. 2011;12:245

43. Ewing B, Hillier L, Wendl MC, Green P. Base-calling of automated sequencer traces using phred. I. Accuracy assessment. Genome Res. 1998;8(3):175-85.

44. Ewing B, Green P. Base-calling of automated sequencer traces using phred. II. Error probabilities. Genome Res. 1998;8(3):186-94.

45. Gordon D, Abajian C, Green P. Consed: a graphical tool for sequence finishing. Genome Res. 1998;8(3):195-202.

46. Tang B, Wang Q, Yang M, Xie F, Zhu Y, Zhuo Y, et al. ContigScape: a Cytoscape plugin facilitating microbial genome gap closing. BMC Genomics. 2013;14:289

47. Lukashin AV, Borodovsky M. GeneMark.hmm: new solutions for gene finding. Nucleic Acids Res. 1998;26(4):1107-15.

48. Delcher AL, Phillippy A, Carlton J, Salzberg SL. Fast algorithms for large-scale genome alignment and comparison. Nucleic Acids Res. 2002;30(11):2478-83.

49. Zhang $C T$, Wang J. Recognition of protein coding genes in the yeast genome at better than 95\% accuracy based on the Z curve. Nucleic Acids Res. 2000;28(14):2804-14

50. Marchler-Bauer A, Anderson JB, Cherukuri PF, DeWeese-Scott C, Geer LY, Gwadz M, et al. CDD: a Conserved Domain Database for protein classification. Nucleic Acids Res. 2005;33(Database issue):D192-196

51. Finn RD, Mistry J, Tate J, Coggill P, Heger A, Pollington JE, et al. The Pfam protein families database. Nucleic Acids Res. 2010:38(Database issue):D211-222.

52. Lowe TM, Eddy SR. tRNAscan-SE: a program for improved detection of transfer RNA genes in genomic sequence. Nucleic Acids Res. 1997;25(5):955-64

53. Li SJ, Zhang CC, Li XW, Tian KC, Tang GP, Wang DM, et al. Molecular typing of Leptospira interrogans strains isolated from Rattus tanezumi in Guizhou Province, Southwest of China. Biomed Environ Sci. 2012;25(5):542-8.

54. Livak KJ, Schmittgen TD. Analysis of relative gene expression data using real-time quantitative PCR and the 2(-Delta Delta C(T)) Method. Methods. 2001;25(4):402-8
55. Schmittgen TD, Zakrajsek BA, Mills AG, Gorn V, Singer MJ, Reed MW Quantitative reverse transcription-polymerase chain reaction to study mRNA decay: comparison of endpoint and real-time methods. Anal Biochem. 2000;285(2):194-204.

56. Cai CS, Zhu YZ, Zhong $Y$, Xin XF, Jiang XG, Lou XL, et al. Development of O-antigen gene cluster-specific PCRs for rapid typing six epidemic serogroups of Leptospira in China. BMC Microbiol. 2010;10:67.

\section{Submit your next manuscript to BioMed Central and take full advantage of:}

- Convenient online submission

- Thorough peer review

- No space constraints or color figure charges

- Immediate publication on acceptance

- Inclusion in PubMed, CAS, Scopus and Google Scholar

- Research which is freely available for redistribution 